\title{
Influence of the thermal contact resistance in current-induced domain wall depinning
}

\author{
Cristina López ${ }^{1}$, Eduardo Ramos ${ }^{1}$, Manuel Muñoz ${ }^{2}$, S. Kar-Narayan³ , N. D. \\ Mathur $^{3}$ and José L. Prieto ${ }^{1}$ \\ ${ }^{1}$ Instituto de Sistemas Optoelectrónicos y Microtecnología (ISOM), Universidad \\ Politécnica de Madrid, Avda. Complutense 30, 28040 Madrid, Spain. \\ ${ }_{2}^{2}$ IMM-Instituto de Microelectrónica de Madrid (CNM-CSIC), Isaac Newton 8, PTM, \\ 28760 Tres Cantos, Madrid, Spain \\ ${ }^{3}$ Materials Science, University of Cambridge, Cambridge CB3 OFS, United Kingdom \\ E-mail: cristina.lopez@isom.upm.es
}

\begin{abstract}
In this work we study the influence of the thermal contact resistance on the temperature of a typical nanostripe used in current induced magnetic domain wall movement or depinning. The thermal contact resistance arises from an imperfect heat transport across the interface between the metallic ferromagnetic nanostripe and the substrate. We show that this parameter, which is likely nonzero in any experimental device, increases the temperature in the nanostripe considerably. When the current is injected in the nanostripe in nanosecond long pulses, the larger temperature also implies a reduction of the effective current density delivered by the pulse generator. Both, the thermal contact resistance and the dynamic response of the pulse generator, are usually neglected in theoretical estimations of the influence of spin transfer torque on domain wall displacement and depinning. Here we show that only if the thermal contact resistance and the electric resistivity of the ferromagnetic nanostripe are optimized to the best values reported in the bibliography, the Joule heating may not be so crucial for current densities of the order of $10^{8} \mathrm{~A} / \mathrm{cm}^{2}$. Also, the use of physical constrictions (notch) to pin the magnetic domain wall may complicate the interpretation of the results as they always come together with relevant thermal gradients.
\end{abstract}

\section{Introduction}

The study of the pinning and depinning of magnetic domain walls (DW) in ferromagnetic nanostripes has been a very popular subject of research since the introduction of the concept of the race-track memory just over a decade $\mathrm{ago}^{1}$. In particular, the use of electric current to achieve the DW depinning via spin transfer torque ${ }^{2}$ has been the quintessential process in these type of experiments ${ }^{3,4}$, as it would set a path for the race-track memories to function without coils or external magnetic fields.

In a current-induced experiment, a typical device would be a ferromagnetic nanostripe of about few hundred nanometres wide and few nanometres thick, with a geometrical constriction in the middle (notch) to pin the DW. An electric current is delivered to the nanostripe to achieve DW displacement, DW depinning from the notch or even a DW transformation via spin transfer torque. The electric current can be DC but it is often delivered by pulses in the nanosecond range, due to the large current density required for spin transfer torque experiments, typically of the order of $10^{8} \mathrm{~A} / \mathrm{cm}^{2}$. This large current density comes necessarily together with a detrimental Joule heating that could cause any of the above effects on the DW or even the destruction of the device. Heat in the magnetic nanostripe can bring local areas to a temperature close to the Curie temperature of the ferromagnetic material or create thermal gradients that may contribute to the movement of the $\mathrm{DW}^{5}$. This makes the correct interpretation of the results difficult.

Few of the early experiments dealing with currentinduced DW movement or depinning estimated experimentally the temperature of a ferromagnetic 
nanostripe when a large DC current density was flowing through $\mathrm{it}^{6}$, or when the current was delivered in long ${ }^{7,8}$ or short (nanosecond) pulses ${ }^{9}$. The different substrates and dimensions of the nanostripes in these studies made the comparison of these results difficult. More recent studies used numerical methods to make an accurate estimation of the temperature in the different areas of the nanostripe ${ }^{5}$ and, importantly, close to the notch were the DW is pinned ${ }^{10}$. Last year, a couple of reports merged micromagnetic simulations with the heat transport so the magnetization dynamics of previous experiments ${ }^{11,12}$ could be accurately described. These works highlighted the importance of thermal gradients in experiments dealing with currentinduced DW depinning ${ }^{3}$ or displacement ${ }^{5}$, to the point that the contribution of spin transfer torque was found almost negligible in comparison to the thermal contribution.

Recently we published a work where values of the temperature in ferromagnetic nanostripes obtained experimentally were fitted to COMSOL simulations ${ }^{13}$. Reference 13 highlighted the importance of using experimental realistic values for the resistivity of the nanostripe and its dependence with temperature. We also introduced a non-zero value of the interface thermal contact resistance, between the nanostripe and the substrate. The thermal contact resistance arises from a non-perfect phonon transport across an interface separating two different materials or separating the same material but with different structure. In Ref. 13, by comparing our estimations of temperature in the nanostripe with previous experimental results, we inferred that the nanostripes used in most experiments must have a non-zero value of the thermal contact resistance and, in most cases, a value quite similar to the one we estimated in Ref. 13.

The thermal contact resistance between the ferromagnetic nanostripe and the substrate has been always assumed to be zero in works dealing with current-induced domain wall depinning and it is still assumed to be zero in recent reports ${ }^{11,12}$. In this work, with the help of COMSOL, we show that, even for the smallest values of the thermal contact resistance, the temperature in both the stripe and the notch is considerably higher than when this parameter is considered zero. This implies an underestimation of the thermal contribution with respect to the contribution of the spin transfer torque in previous experiments. Our results could give an explanation of why current densities larger than $10^{8} \mathrm{~A} / \mathrm{cm}^{2}$ can rarely be delivered to the nanostripe without destruction of the device ${ }^{14}$, even if nanosecond long pulses are used. Here we also analyse the influence of the electrical resistivity of the ferromagnetic material as a possible mean to reduce the Joule heating. With the results obtained, we analyse the effect of reducing the pulse length as a possible strategy to reduce the Joule heating. We discuss the problem in the light of several parameters such as the thermal contact resistance, the resistivity of the nanostripe and the thermal conductivity of the substrate.

We come to the general conclusion that Joule heating is going to be very relevant even for small (but non-zero) values of thermal contact resistance and that, the thermal gradients around the notch, would be very large unless the notch is very shallow, which would compromise its ability to pin the DW reliably. We argue that the traditional geometry of a nanostripe (with or even without a notch) may not be the best experimental approach to explore the influence of spin transfer torque on a DW. This conclusion has been perhaps naturally assumed by the scientific community and other approaches for the manipulation of DWs in nanodevices via electric current are now more popular, such as the use of materials with anisotropy perpendicular to plane ${ }^{15}$, or the use of torques arising from spin-orbit interactions with adjacent heavy non-magnetic metals ${ }^{16}$.

\section{Description of the experiment}

Most of the results described in this work are based on simulations performed for a nanostripe modelled $5 \mu \mathrm{m}$ long and $300 \mathrm{~nm}$ wide, with a triangular notch in the middle, $100 \mathrm{~nm}$ deep and $300 \mathrm{~nm}$ wide, as shown in figure 1a. For the simulations, the nanostripe is considered to be mainly Permalloy (Py) with the structure $\mathrm{Pt}(1) / \mathrm{Ta}(2) / \mathrm{Ni}_{80} \mathrm{Fe}_{20}(10) / \mathrm{Ta}(2)$, with the numbers between brackets indicating the thickness of each layer in nanometers and it is deposited over a $\mathrm{Si}$ substrate, with a thermal oxide layer of $25 \mathrm{~nm}$. This $\mathrm{SiO}_{2}$ layer constitutes a large thermal resistance but, experimentally, it is usually required to avoid current leaking through the substrate when pulses of few nanoseconds are delivered to the nanostripe.

When a current pulse is sent from the pulse generator to the magnetic nanostripe, a heat power per unit of volume is generated by Joule effect in the resistive nanostripe $Q=\rho \cdot j^{2}$, with $\rho$ electric resistivity of the Py and $j$ the current density. This heat increases the temperature of the nanostripe which is also linked to an increase of its electric resistance. The pulse generator responds dynamically to this increase of resistance by delivering less current. The current delivered by the pulse generator at any time (or temperature of the nanostripe) can be extracted easily from the following formula,

$$
I[T(t)]=\frac{2 V_{p}}{R_{n s}(T)+2 Z_{0}}
$$


where Rns $(T)$ is the resistance of the nanostripe, which is temperature dependent, $Z_{0}$ is usually $50 \Omega$ and $V_{p}$ is the voltage amplitude of the pulse. The Joule heating is dissipated entirely by thermal conduction to the $\mathrm{Si} / \mathrm{SiO}_{2}$ substrate, as other mechanisms of heat dissipation, such as convection or radiation, are negligible for nanostripes with such a small dissipating surface. The temperature profile in the nanostripe and the substrate follows the heat diffusion equation,

$$
\nabla^{2} T+\frac{Q}{k}=\frac{C_{V}}{k} \frac{\partial T}{\partial t}
$$

with $T$ the temperature, $k$ the thermal conductivity, $C_{V}$ the heat capacity per unit of volume and $Q=\rho \cdot j^{2}$ the heating term. This equation has been solved with COMSOL, using typical parameters for these materials $^{5,10}$, for Permalloy $C_{V}=3.7 \cdot 10^{6} \mathrm{~J} / \mathrm{m}^{3}, k=46.4$ $\mathrm{W} / \mathrm{m} \cdot \mathrm{K}$ and for the Si substrate $C_{V}=1.6 \cdot 10^{6} \mathrm{~J} / \mathrm{m}^{3}$ and $k=130 \mathrm{~W} / \mathrm{m} \cdot \mathrm{K}$. For the $\mathrm{SiO}_{2}$ layer we have used a temperature dependent thermal resistance, extracted from COMSOL $^{17}$ (see also Fig. 2c in Ref.13). In figure $1 \mathrm{~b}$ we plot the temperature profile in the nanostripe along a longitudinal line adjacent to the tip of the triangular notch (dashed white line in figure 1a), at the end of a $2.5 \mathrm{~V}, 100 \mathrm{~ns}$ pulse, which corresponds to a current density of $0.9 \cdot 10^{8} \mathrm{~A} / \mathrm{cm}^{2}$. For this simulation, we have used the electric resistivity obtained experimentally for the Permalloy we deposit in our laboratory, patterned in the dimensions of the nanostripe, $\rho(T)=0.092 T+34.8 \mu \Omega \cdot \mathrm{cm}$. At $300 \mathrm{~K}$, the resistivity of our Py is very similar to the one used and measured by other authors ${ }^{18}$ (see also table in Ref.10). The temperature dependence above the Curie temperature of Py has been extrapolated from Ref. 19 , as also shown in figure $2 b$ in our previous work $^{13}$. For the curve shown in figure $1 b$, we have also added a thermal contact resistance between the Py and the substrate of $2 \cdot 10^{-8} \mathrm{~m}^{2} \mathrm{~K} / \mathrm{W}$.
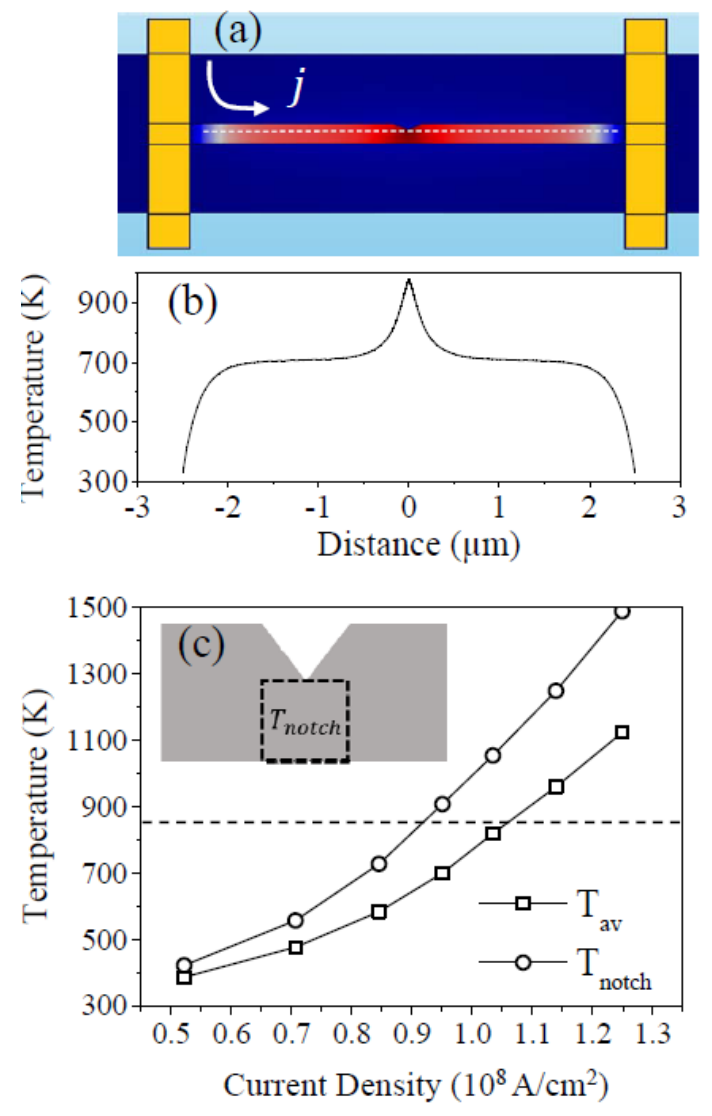

Figure 1. (a) Schematic diagram of the simulated stripe. (b) Temperature profile along a horizontal line parallel to the stripe indicated by a white dashed line, when a pulse of $2.5 \mathrm{~V}$ is delivered to the device. (c) Average temperature in the stripe and temperature in the notch for different current densities as explained in the text. The inset to figure 1c shows the box where the temperature at the notch is calculated as an average temperature in that region.

Figure $1 \mathrm{~b}$ shows that the temperature in the tip of the notch is considerably higher than in the rest of the nanostripe, due to the larger current density in that area. The tip temperature ( $x=0$ in figure $1 b)$ is not representative of the realistic average temperature in the notch though, as we will experimentally justify bellow. Therefore, we will consider the temperature in the notch $T_{\text {notch }}$ as the average temperature in the area highlighted with a dashed square in the inset to figure 1c. Figure 1c shows the average temperature $T_{a v}$ (i.e. the temperature averaged across the entire nanostripe) and the temperature in the notch $T_{\text {notch }}$, for different current densities. Noticeably, even for a current density of $\sim 0.9 \cdot 10^{8} \mathrm{~A} / \mathrm{cm}^{2}$ the temperature in the notch is very close to the Curie Temperature of Py $(\sim 850 \mathrm{~K})$.

Performing a quantitative experimental determination of the temperature in the notch is very challenging. On the other hand, a qualitative measurement of the distribution of temperatures around the notch may be sufficient to give us a 
valuable indication of the merits of the COMSOL simulations in some aspects. Figure 2 shows an Atomic Force Microscopy image (Veeco AFM) (Fig. 2a) and a thermal image, taken by Scanning Thermal Microscopy (SThM) (Fig. 2b) of a Py nanostripe when a DC current density of $3.5 \cdot 10^{7} \mathrm{~A} / \mathrm{cm}^{2}$ flows through it. This current density is the maximum that could be applied without destruction of the device. The AFM model was a Bruker Dimension AFM, with a SThM module from Anasys Instruments. The thermal image is taken with a Pt track on an AFM tip, this track constituting an arm from a Platinum Wheatstone bridge. When the Pt at the AFM samples a hot surface, it unbalances the Wheatstone bridge and a voltage can be measured. This is mainly a qualitative measurement as it is very difficult to calibrate the heat diffused from the device to the AFM tip and how the 3D distribution of the heat diffused to the tip, affects the voltage induced at the Wheatstone bridge.

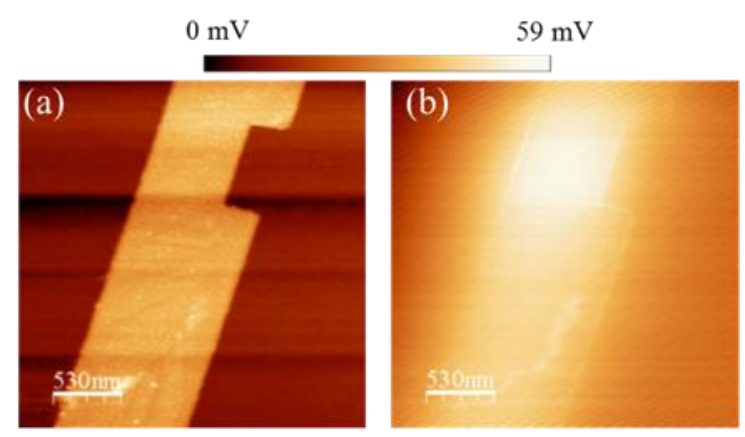

Figure 2. AFM (a) and thermal image (b) of a nanostripe with a square notch when a DC current density of $3.5 \cdot 10^{11} \mathrm{~A} / \mathrm{cm}^{2}$ is flowing through the stripe. Any further increase of the current density would cause destruction of the device. Temperature is measured by a Pt track on an AFM tip, this track constitutes one arm of a Wheatstone bridge. This is a qualitative measurement due to the difficulties related to the calibration of the heat diffused to the bulk of the AFM tip.

We could not manage to measure many devices with this technique. The scan has to be very slow (1 $\mathrm{Hz}$ per scan) to allow the AFM tip to heat up and reach a steady state. Therefore, many devices would get destroyed while rising the current density before we could obtain an image. Figure $2 b$ shows one of our successful attempts to image the notch area just before the breakdown of the device. In the $1 \mu \mathrm{m}$ wide, $10 \mathrm{~nm}$ thick Py nanostripe imaged in figure 2, the width of the stripe was decreased $700 \mathrm{~nm}$ with a square notch. Therefore the maximum DC current density that the notch area could withstand was on the order of $5 \cdot 10^{7} \mathrm{~A} / \mathrm{cm}^{2}$.

The first thing that one can notice looking at figure $2 \mathrm{~b}$ is that the temperature in the notch area is quite uniform and there are no strong hot points at the edges of the notch. This justifies our approach for averaging the temperature in the notch area in our simulations as we show in figure 1c.

Also, the lateral thermal gradients around the notch become quite obvious in figure $2 \mathrm{~b}$. The notch is clearly hotter than the rest of the nanostripe even only few hundreds of nanometers away from the notch. We cannot know the temperature of the notch in figure $2 b$ but with the scale provided, the temperature seems to drop about $20 \%$ in only one micrometer. This nanostripe was deposited over a $\mathrm{Si}$ substrate with a thin (few nanometers) oxide layer. As the Py is a good thermal conductor due to its metallic nature, this large lateral thermal gradients can only be explained if the substrate cannot dissipate the heat well. As we argued in our previous work $^{13}$, a large lateral thermal gradient on a nanostripe deposited over a thermally conductive substrate such as $\mathrm{Si}$, is a sign of the presence of a large thermal contact resistance.

Unfortunately, getting an actual value of thermal contact resistance form a qualitative image such as the one in figure $2 \mathrm{~b}$ is very difficult. We have no knowledge of the temperature at which the stripe breaks down. Nevertheless, as it will become clearer with the simulations presented in the following sections, the $20 \%$ variation between the temperature at the notch and the main temperature of the stripe shown experimentally in figure $2 b$, could easily lead to values ranging from 2 to $5 \cdot 10^{8} \mathrm{~m}^{2} \mathrm{~K} / \mathrm{W}$ (or even higher) for the thermal contact resistance.

The fact that the nanostripe is hotter around the notch has two main implications. Firstly, the large thermal gradients along the nanostripe can easily be the main contribution to the movement and depinning of the DW as it has been shown recently ${ }^{11,20}$. Secondly, a large buildup of temperature around the notch would likely cause the destruction of the device. We may compare the results in figure $1 \mathrm{c}$ with the results displayed in figure $3 c$ of Ref.12. For a given current density, we obtain considerably higher temperature in the notch ( $\left.\mathrm{T}_{\text {notch }}\right)$ and in the stripe $\left(\mathrm{T}_{\mathrm{av}}\right)$. There are two reasons for this large difference. On one hand, we have used a $25 \mathrm{~nm}$ thick $\mathrm{SiO}_{2}$ layer between the nanostripe and the Si substrate, rather than a thin native oxide layer. As stated above, the $25 \mathrm{~nm}$ thick $\mathrm{SiO}_{2}$ layer constitutes a large thermal resistance but it is required to avoid current leaking through the substrate when pulses of few nanoseconds are delivered to the nanostripe ${ }^{21}$. Additionally, and more importantly, for the simulation displayed in figure 1c we have used a $2 \cdot 10^{-8} \mathrm{~m}^{2} \mathrm{~K} / \mathrm{W}$ thermal contact resistance, while the authors of Ref. 12 assumed this value to be zero. 
In this work we have delivered the current in 100ns long pulses to be consistent with our previous experimental calibration ${ }^{13}$. Note that, if very short pulses are used (only a few nanoseconds long) the temperature in the nanostripe is not as high as it is after a $100 \mathrm{~ns}$ pulse. Nevertheless, as it is discussed in more detail in the conclusions, the temperature builds up very quickly in the very first nanoseconds of the pulse $\mathrm{s}^{12,13,22}$ and the main conclusions of the work still stand. For the substrate used here ( $\mathrm{Si}$ with $25 \mathrm{~nm}$ thick thermal $\mathrm{SiO}_{2}$ ), after $100 \mathrm{~ns}$ of current pulse, the current density and the temperature in the nanostripe have almost reached the steady state. This allows us to plot the results against the current density at the end of the 100ns pulse, rather than against the voltage of the pulse, which would make the comparison with previous results more difficult.

\section{A. Influence of the thermal contact resistance}

Experimentally, the thermal contact resistance between the nanostripe and the substrate is likely unavoidable. The ferromagnetic nanostripe has different crystalline structure than the substrate and their chemical structure is also different. Therefore the phononic heat transport across the interface is not going to be perfect, leading to a non-zero interfacial thermal contact resistance $R_{\text {int }}$ (see schematic representation on top of figure 3 ).

In order to visualize how important the contact thermal resistance is, we can compare it to the resistance of a layer of $\mathrm{SiO}_{2}$. The one dimensional heat flow in steady state is described by the Fourier law,

$$
q=-k A \frac{\partial T}{\partial x}
$$

where $q$ is the heat flow (in Watts), $k$ the thermal conductivity and $A$ the area. In an infinite plane with the heat flowing perpendicular to the plane, the equation can be rewritten as,

$$
q=-k A \frac{\Delta T}{\Delta x}
$$

The thermal resistance of the plane is therefore defined as $\Delta x / k A$, with $\Delta x$ the thickness of the plane. For instance, a $25 \mathrm{~nm}$ thick layer of $\mathrm{SiO}_{2}$ with a room temperature thermal conductivity of $k=1 \mathrm{~W} / \mathrm{m} \cdot \mathrm{K}$, would constitute a thermal resistance of $2.5 \cdot 10^{-8} \mathrm{~m}^{2} \mathrm{~K} / \mathrm{W}$ per square meter. With this quick calculation one can easily realize how relevant a contact thermal resistance of for instance $2 \cdot 10^{-8}$ $\mathrm{m}^{2} \mathrm{~K} / \mathrm{W}$ can be, as it is effectively equivalent to a 20 $\mathrm{nm}$ thick layer of $\mathrm{SiO}_{2}$. As we showed in our previous work $^{13}$, it is possible to infer the value of the interfacial thermal contact resistance by fitting COMSOL simulations to experimental measurements of the dynamic thermal response of nanostripes with different widths, when injecting current pulses in the nanosecond range. The rise time of the temperature and the final steady state temperature cannot both be fitted for the different widths of the nanostripes unless the correct value of the contact thermal resistance is used (see also conclusions).

Careful engineering of the buffer layers can minimize the contact thermal resistance. The smallest interface contact resistance estimated from the data reported in previous studies is around $1 \cdot 10^{8}$ $\mathrm{m}^{2} \mathrm{~K} / \mathrm{W}$ obtained using a $\mathrm{Fe} / \mathrm{AlO}_{\mathrm{x}}$ buffer in Ref. 3, although values close to $5-6 \cdot 10^{-8} \mathrm{~m}^{2} \mathrm{~K} / \mathrm{W}$ may be more common ${ }^{13}$. It is important therefore to draw some attention to the effects of the interfacial thermal contact resistance on the temperature of the nanostripe.

Figure 3 shows the temperature in the notch and the average temperature in the entire stripe, versus the current density for different values of the thermal contact resistance. Note again that the current density is the value at the end of the $100 \mathrm{~ns}$ pulse, which is close to the final steady state value but considerably smaller than the current density in the first instants of the pulse before the heat starts to build up.
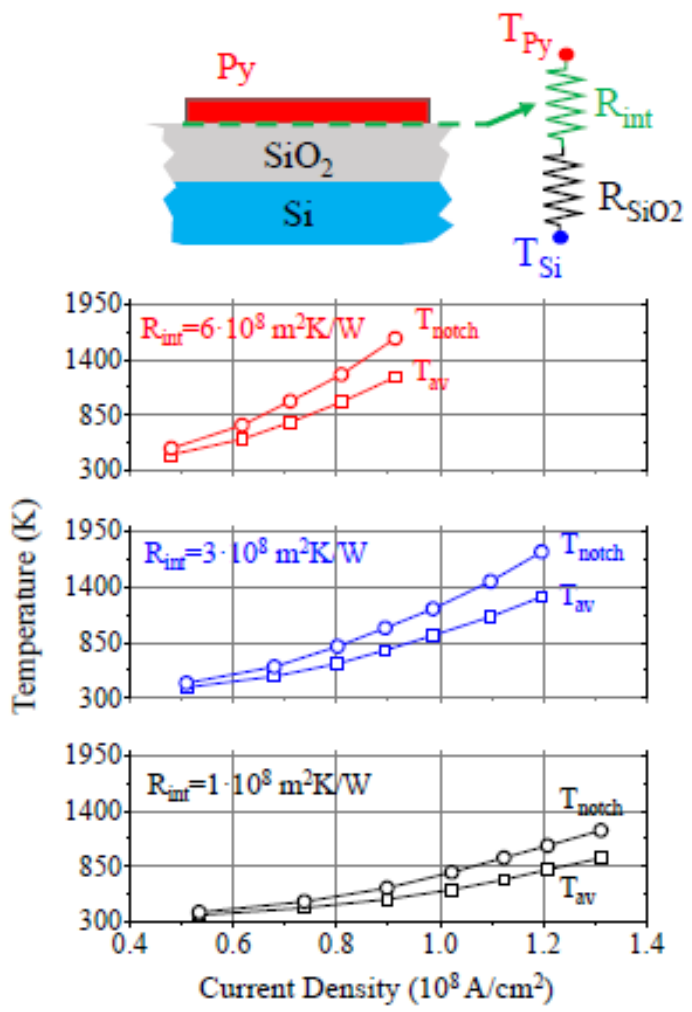

Figure 3. Top figure, schematic representation of the thermal contact resistance between the ferromagnetic nanostripe and the $\mathrm{SiO}_{2}$ layer. Bottom figures, average temperature in the nanostripe $\left(T_{a v}\right)$ and temperature in the notch area $\left(T_{\text {notch }}\right)$ for three different values of the thermal contact resistance $\left(R_{\text {int }}\right)$.

For a $R_{\text {int }}=3 \cdot 10^{-8} \mathrm{~m}^{2} \mathrm{~K} / \mathrm{W}$ and a current density of $j=10^{8} \mathrm{~A} / \mathrm{cm}^{2}$ the average temperature in the stripe is 
already over the Curie temperature of Py and the temperature in the notch is over $1000 \mathrm{~K}$. For $R_{\text {int }}$ $=6 \cdot 10^{-8} \mathrm{~m}^{2} \mathrm{~K} / \mathrm{W}$, the temperature for current densities close to $10^{8} \mathrm{~A} / \mathrm{cm}^{2}$ is so high that one would not expect the nanostripe to hold that far and it would likely be destroyed for smaller current densities. This may explain why there are not many research groups that manage to inject current densities higher than $10^{8} \mathrm{~A} / \mathrm{cm}^{2}$ unless they use very short pulses (see also the discussion in conclusions).

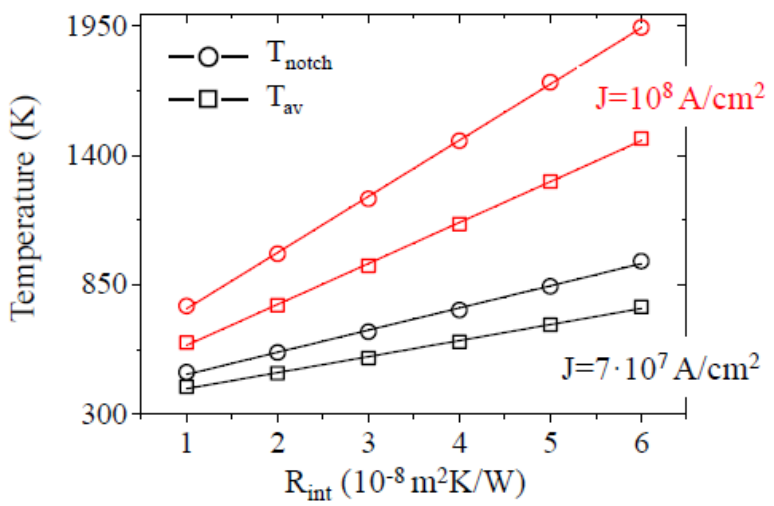

Figure 4. Temperature in the notch and average temperature in the entire stripe versus the thermal contact resistance, for two selected current densities.

Figure 4 shows $T_{\text {notch }}$ and $T_{a v}$ for two selected current densities, plotted versus the interface thermal contact resistance $R_{\text {int }}$. It becomes clear again that, even for moderate current densities, unless the thermal contact resistance is quite low, the temperature builds up very quickly.

In figure 4 the temperature grows almost linearly with $R_{\text {int }}$. Also, as shown in figure 3 , the temperature is quadratic with the current density, as expected from Joule heating. Therefore, by running a number of simulations, we can deduce a functional dependency to describe the whole range under study. The temperature $T$ versus current density $j$ follows the expression $T(j)=a \cdot j^{2}+b \cdot j+c$, with $a, b$ and $c$ dependent on the thermal contact resistance through the general cubic function, $m \cdot R_{\text {int }}^{3}+n$. $R_{\text {int }}^{2}+o \cdot R_{\text {int }}+p$. The values for the constants $m$, $n, o$ and $p$ are given in Table I for both $T_{\text {notch }}$ and $T_{a v}$, using $j$ in units of $10^{8} \mathrm{~A} / \mathrm{cm}^{2}$ and $R_{\text {int }}$ in units of $10^{-8}$ $\mathrm{m}^{2} \mathrm{~K} / \mathrm{W}$.

\begin{tabular}{lllll}
\hline & $M$ & $N$ & $O$ & $P$ \\
\hline$A$ & 0 & 0 & 253.1 & 450.3 \\
\hline$B$ & 6.64 & -67.4 & 116.9 & -651 \\
\hline$C$ & -2.39 & 25.6 & -81.6 & 539.5 \\
\hline$A$ & -18.3 & 169.3 & -48.4 & 762.4 \\
\hline$B$ & 25.1 & -226.5 & 390.8 & -718.7 \\
\hline$C$ & -8.3 & 76.2 & -169.3 & 526.8 \\
\hline
\end{tabular}

Table I. Values for the constants that fit the temperatures obtained in the simulations to the expressions described in the text, as a function of the thermal contact resistance.

The maximum deviation between the result obtained with these expressions and the result obtained with the simulations is about $5 \%$. This maximum deviation is obtained at the extreme temperatures in the range under study (either low or high) and therefore, not very relevant for the main conclusions. With the expressions obtained from Table I, we can plot all the range under study in a color contour map for both $T_{n o t c h}$ and $T_{a v}$, as shown in figure 5 . The dashed line in figure 5 indicates the $850 \mathrm{~K}$ contour line, the Curie temperature of Permalloy. At a glance one can see that, unless the thermal contact resistance is unrealistically low, the thermal gradient around the notch is likely the main player in any experiment dealing with domain wall depinning by spin transfer torque.

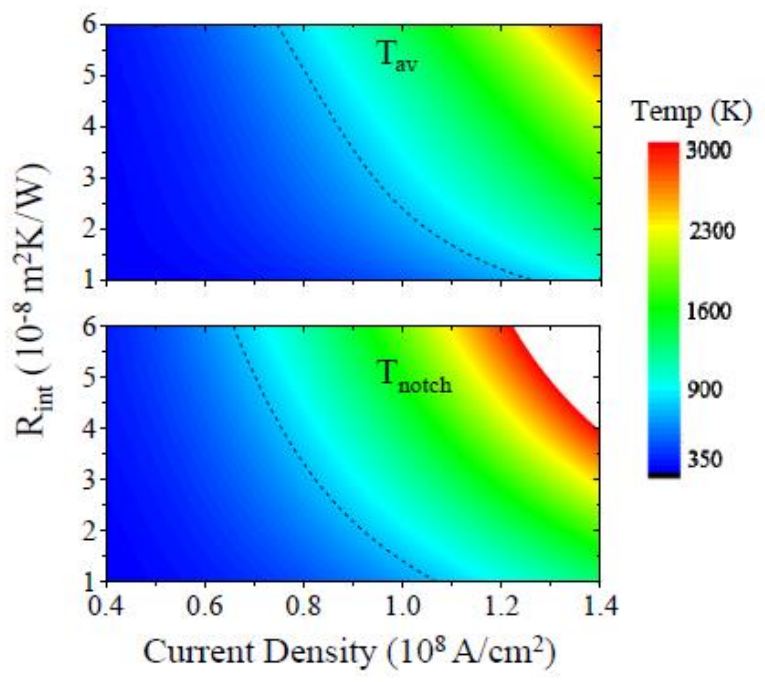

Figure 5. Contour colour map for the average temperature and the notch temperature versus current density and thermal contact resistance. The dashed line indicates the Curie temperature of Py.

\section{B. Influence of the resistivity of Permalloy}

The heating term in equation [1], $Q=\rho \cdot j^{2}$, depends also on the resistivity of Py, so one should be able to deliver more current density to the device by depositing a low resistive Py layer. The Room Temperature (RT) resistivity of Py used in the simulations above is $62 \mu \Omega \cdot \mathrm{cm}$, measured in patterned devices (rather than in films). Values between 25 and $65 \mu \Omega \cdot \mathrm{cm}$ have been reported ${ }^{23,24}$ for Py films deposited at a specific substrate temperature. Therefore, we have run a set of simulations fixing the contact resistance to a medium-low value of $2 \cdot 10^{-8} \mathrm{~m}^{2} \mathrm{~K} / \mathrm{W}$, and changing the Py RT resistivity in each simulation (for simplicity, we have assumed always the same 
temperature dependence of the resistivity, $0.092 T$ in $\mu \Omega \cdot \mathrm{cm})$. As before, we can fit the simulations to a quadratic expression $T(J)=a \cdot j^{2}+b \cdot j+c$, where $a, b$ and $c$ depend now linearly on the RT resistivity of Py $\left(\rho_{R T}\right)$ like $m \cdot \rho_{R T}+n$. The different values for these constants can be found in Table II, using $j$ in units of $10^{8} \mathrm{~A} / \mathrm{cm}^{2}$ and $\rho_{R T}$ in units of $\mu \Omega \cdot \mathrm{cm}$.

\begin{tabular}{cccc}
\hline & $m$ & $n$ & \\
\hline$A$ & 5.1 & 638.4 & \\
\cline { 1 - 3 }$b$ & 4.11 & -886.3 & \\
\cline { 1 - 3 }$c$ & -1.7 & 563 & \\
\hline$a$ & 6.57 & 808.4 & \\
\hline$b$ & 3.62 & -861.6 & \\
\hline$c$ & 5.2 & 282 & -0.05 \\
& & & $\cdot \rho_{R T}^{2}$ \\
\hline
\end{tabular}

Table II. Values for the constants that fit the temperatures obtained in the simulations to the expressions described in the text, as a function of the RT resistivity of Py.

With these data we can build the contour maps displayed in figure 6. As expected the resistivity of Py is not as influential as the thermal contact resistance in the calculation of the final temperature of the nanostripe (figure 5). For example, if for a stripe with a medium RT resistivity of $50 \mu \Omega \cdot \mathrm{cm}$ $\left(R_{\text {int }}=2 \cdot 10^{-8} \mathrm{~m}^{2} \mathrm{~K} / \mathrm{W}\right)$ the notch reaches the Curie temperature for $j=10^{8} \mathrm{~A} / \mathrm{cm}^{2}$, by reducing the resistivity to the lowest reported, $25 \mu \Omega \cdot \mathrm{cm}$, the notch would reach the Curie temperature for $j=1.2 \cdot 10^{8} \mathrm{~A} / \mathrm{cm}^{2}$, which is only a moderate improvement.

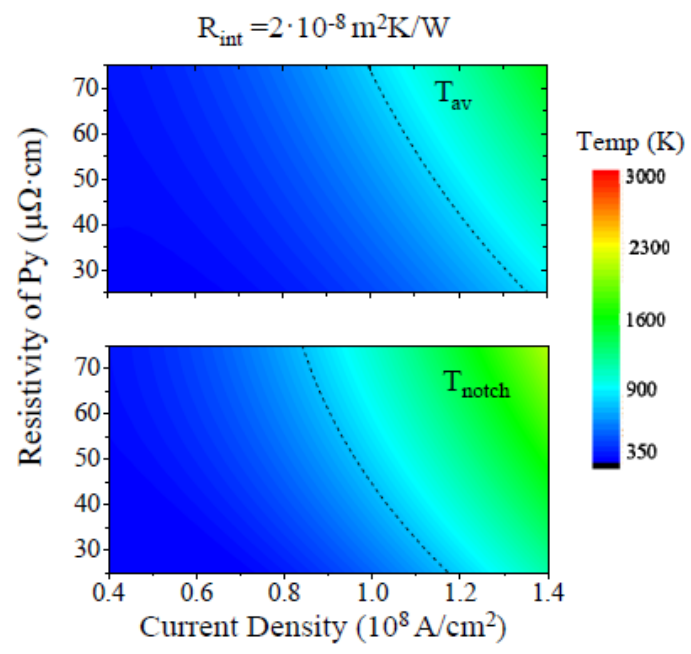

Figure 6. Contour colour map for the average temperature and the temperature in the notch versus current density and RT resistivity of the Py nanostripe, for a fixed thermal contact resistance of $2 \cdot 10^{-8} \mathrm{~m}^{2} \mathrm{~K} / \mathrm{W}$. The dashed line indicates the Curie temperature of $\mathrm{Py}$.
Figure 5 and 6 show that the Joule heating is going to be the dominant force transforming and/or moving a magnetic domain wall pinned at the notch, for the dimensions of the nanostripe and notch described in this work, unless the thermal contact resistance and the resistivity of the Py are very low. Reducing the thickness of Py may make the situation a bit better, as a smaller cross section leads to a smaller volume generating heat $\left(Q=\rho \cdot j^{2}\right.$ is heat per unit of volume). Again, a quick calculation shows that, unless the thermal contact resistance is very low, the Joule heating is still going to be too high to perform an accurate evaluation of the contribution of spin transfer torque.

A possible solution could be to reduce the depth of the notch. A smaller constriction would imply that $T_{\text {notch }}$ would tend to $T_{a v}$. Table III shows the effect of changing the dimensions of the triangular notch for a fixed current density of $10^{8} \mathrm{~A} / \mathrm{cm}^{2}$ and a low thermal contact resistance of $2 \cdot 10^{-8} \mathrm{~m}^{2} \mathrm{~K} / \mathrm{W}$. In all cases, $T_{a v}$ results in $770 \pm 10 \mathrm{~K}$.

\begin{tabular}{lccccc}
\hline $\begin{array}{l}\text { Width } \\
(\mathrm{nm}) \rightarrow\end{array}$ & 100 & 200 & 300 & 400 & 500 \\
$\begin{array}{l}\text { Depth }(100 \\
\mathrm{nm})\end{array}$ & & & & & \\
\hline$T_{\text {notch }}(\mathrm{K}) \rightarrow$ & 943 & 969 & 982 & 1000 & 1008 \\
\hline $\begin{array}{l}\text { Depth } \\
(\mathrm{nm}) \rightarrow\end{array}$ & 50 & 100 & 150 & 200 & \\
$\begin{array}{l}\text { Width }(300 \\
\text { nm) }\end{array}$ & & & & & \\
\hline$T_{\text {notch }}(\mathrm{K}) \rightarrow$ & 790 & 982 & 1226 & 1700 & \\
\hline
\end{tabular}

Table III. Temperature in the notch for different dimensions of the notch for a fixed current density of $10^{8} \mathrm{~A} / \mathrm{cm}^{2}$ and a fixed thermal contact resistance of $2 \cdot 10^{-8} \mathrm{~m}^{2} \mathrm{~K} / \mathrm{W}$, changing the width for a constant depth of $100 \mathrm{~nm}$ (top) and changing the depth for a constant width of $300 \mathrm{~nm}$ (bottom).

By looking at Table III, it becomes clear that, unless the notch is very shallow (50 $\mathrm{nm}$ or less), the thermal gradient around the notch would be quite relevant. Making the notch wider (smoother) only increases $T_{\text {notch }}$ as there is a larger portion of the nanostripe subject to a larger current density. Making the notch narrower than $100 \mathrm{~nm}$ should not make any difference as the current lines cannot follow sharper corners than those in a triagular notch $100 \mathrm{~nm}$ wide and 100nm deep. Therefore, even for a low thermal contact resistance, the thermal gradients around the notch are going to be notorious unless the notch is very shallow, which would sacrifice its pinning reliability.

\section{Conclusions}


As it was shown recently ${ }^{5,21,11}$, heat seems to be the main player in experiments dealing with current induced DW movement or depinning. In previous estimations of the temperature in nanostripes, the thermal contact resistance has been neglected. As we have seen in this article, the temperature for a given current density, can be considerably higher once the thermal contact resistance is taken into account. Therefore, although the significant contribution of the Joule heating is already proven, it may even be more relevant than previously thought.

If a good pulse generator is available, very short pulses can be delivered to the nanostripe in order to reduce Joule heating. Figure 7 shows how the average temperature in the nanostripe rises in the first nanoseconds of a $2 \mathrm{~V}$ pulse, for different thermal contact resistances. On top of each curve, in red font, we display the current density after 10ns of pulse, which is not far from the steady state value. Higher thermal contact resistance would lead to a higher temperature and a slower rise time ${ }^{25}$.

Figure 7 is very interesting for several reasons. Firstly, for good samples with very low thermal contact resistance, the temperature rises very quickly and in about $4 \mathrm{~ns}$ the sample has pretty much reached the steady state. Still, there is a significant rise of the average temperature in the nanostripe for a 'moderate' current density of $0.9 \cdot 10^{8} \mathrm{~A} / \mathrm{cm}^{2}$ for the lowest $R_{\text {int }}=10^{-8} \mathrm{~m}^{2} \mathrm{~K} / \mathrm{W}$. Therefore, in samples with very low thermal contact resistance, it seems that unless an extremely short $1 \mathrm{~ns}$ pulse is delivered, using short pulses is not going to improve the situation. Secondly, for a more realistic higher thermal contact resistance, because of the slower rise time of the temperature, using a shorter pulse can reduce the temperature appreciably. In any case, as it can be seen in the inset to figure 7 , when a 2 ns pulse is used the average temperature in the stripe and the temperature in the notch are still quite high. Also the difference between $T_{\text {notch }}$ and $T_{a v}$ is at least $100 \mathrm{~K}$ in any case, leading to a large thermal gradient around the notch.

Finally, figure 7 includes information of the current density that should not go unnoticed. Each curve is labeled in red with the value of the current density after $10 \mathrm{~ns}$. Noticeably, this value decreases as the thermal contact resistance (and the temperature) increases. This can also be seen in the inset to figure 7, where the current density is represented by red stars (left $y$-axis). As explained in the introduction, when short pulses are used, the pulse generator, which is coupled to a $50 \Omega$ load, responds dynamically to the load resistance. Therefore, when the temperature increases and, with it, the resistance of the stripe, the generator adjusts its output and delivers less current, as shown by formula [1]. This is quite important and it seems to go unnoticed in theoretical estimations of the influence of spin transfer torque. The maximum current density is delivered in the first instants of the pulse, before the temperature builds up considerably. It is in those very first instants when the spin transfer torque would be more relevant. Then, after even only $2 \mathrm{~ns}$, the temperature and the resistance of the stripe have raised considerably and the current density decreases to values that are likely not so relevant for spin transfer torque.

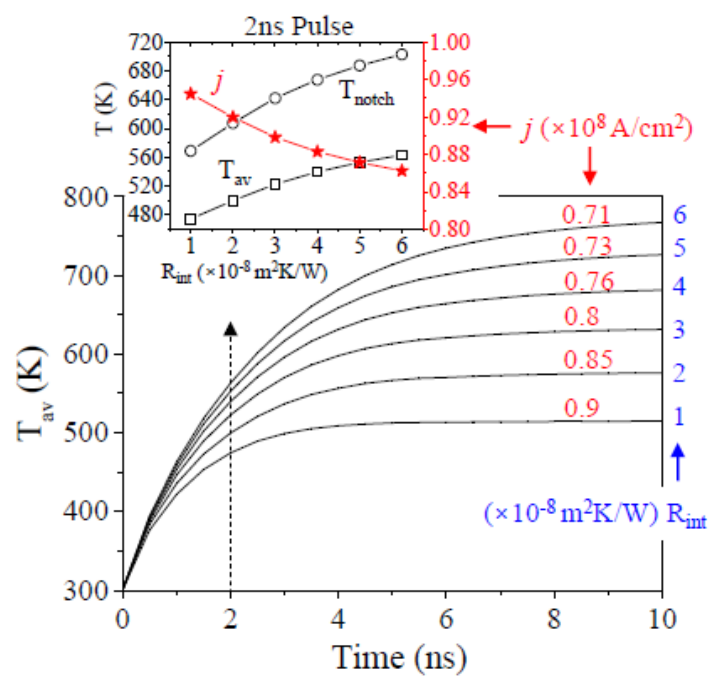

Figure 7. Average temperature in the nanostripe with time after a $2 \mathrm{~V}$ pulse is delivered to the device, for different values of $R_{\text {int }}$ (in blue font on the right hand side of the picture). The current density after $10 \mathrm{~ns}$ of pulse is displayed over each curve in red font. The inset shows the temperature in the nanostripe and in the notch for a $2 \mathrm{~V}$ and $2 \mathrm{~ns}$ pulse and for different values of the thermal contact resistance $R_{\text {int }}$. The current density at the end of the 2 ns pulse for each value of $R_{\text {int }}$ is plotted against the right $y$-axis in red.

Reducing the $\mathrm{SiO}_{2}$ layer over the $\mathrm{Si}$ substrate to less than $25 \mathrm{~nm}$ would improve the thermal dissipation for a fixed thermal contact resistance. In this case though, precautions should be taken when delivering the current in nanoseconds long pulses as the current may partially leak to the Si substrate, especially if the $\mathrm{Si}$ is doped and it has a good electric conductivity. This is usually the case, as it facilitates the e-beam lithography of the nanostripes by avoiding the buildup of charge in the sample during the lithography.

A possible alternative would be the use of a good thermal conductor but electric insulator, such as sapphire or diamond. Figure 8 shows the comparison of the temperature in the nanostripe when using sapphire as a substrate or $\mathrm{Si} / \mathrm{SiO}_{2}(25 \mathrm{~nm})$. Sapphire has a thermal conductivity of $25 \mathrm{~W} / \mathrm{m} \cdot \mathrm{K}$ at RT, which is considerably better than the $1 \mathrm{~W} / \mathrm{m} \cdot \mathrm{K}$ of $\mathrm{SiO}_{2}$. 
As can be seen, using sapphire instead $\mathrm{Si} / \mathrm{SiO}_{2}$ substrate leads to only a small improvement in the temperature for low thermal contact resistance and a marginal improvement for high thermal contact resistance. We have fabricated Py nanostripes on a sapphire substrate with the dimensions and structure described in the introduction. The maximum current density that they can hold in steady state is about $10^{8}$ $\mathrm{A} / \mathrm{cm}^{2}$. For larger current densities, the nanostripes blow up. By looking at figure 8, this experimental fact points towards a medium-low thermal contact resistance. This is a hopeful result and perhaps, with a careful selection of the buffer layers, the use of very good thermal conductors as substrate and very short current pulses, may allow good measurements in this type of experiments.

We can conclude therefore that, unless the nanostripes are engineered so their electric resistivity and their thermal contact resistance with the substrate are both minimized, Joule heating is going to play a very important role in current induced DW movement or depinning. The large temperature (easily close to the Curie temperature of the nanostripe), the thermal gradients and the stochastic nature of the DWs ${ }^{26,27}$, can make the interpretation of the results quite complicated. The thermal contribution is even more relevant when a physical constriction (notch) is used to trap the DW. A possible alternative would be to work in a weak pinning regime (very shallow notches) or using chemical defects to pin the $\mathrm{DW}^{28}$, so there is not an enhanced Joule heating associated to the pinning

\footnotetext{
${ }^{1}$ Parkin S S P, Hayashi M, and Thomas M 2008 Science 320, 190

2 J C Slonczewski 1996 J. Magn. Magn. Mater. 159, L1

${ }^{3}$ M Hayashi, L Thomas, C Rettner, R Moriya, X Jiang, and S S P

Parkin 2006 Phys. Rev. Lett. 97, 207205

${ }^{4}$ M Hayashi, L Thomas, C Rettner, R Moriya, and S S P Parkin 2007

Nature Phys. 3, 21

${ }^{5} \mathrm{~J}$ Torrejon, G Malinowski, M Pelloux, R Weil, A Thiaville, J Curiale, D Lacour, F. Montaigne and M. Hehn, 2012 Phys. Rev. Lett. 109, 106601

${ }^{6} \mathrm{~N}$ Vernier, D A Allwood, D Atkinson, M D Cooke and R P Cowburn 2004 Europhys. Lett. 65, 526

${ }^{7}$ M Laufenberg, W Bührer, D Bedau, P E Melchy, M Kläui, L Vila, G Faini, C A F Vaz, J A C Bland and U Rüdiger 2006 Phys. Rev. Lett. 97, 046602

${ }^{8}$ A Yamaguchi, A Hirohata, T Ono and H. Miyajima 2012 J. Phys.:

Condens. Matter 24, 024201

${ }^{9}$ M Hayashi, PhD thesis 2016 Stanford University

${ }^{10}$ H Fangohr, D S Chernyshenko, M Franchin, T Fischbacher and G

Meier 2011 Phys. Rev. B 84, 054437

${ }^{11}$ V Raposo, S Moretti, M A Hernández and E Martínez 2016 Appl.

Phys. Lett. 108, 042405

12 S Moretti, V Raposo and E Martínez 2016 J. Appl. Phys. 119 213902

${ }^{13}$ E Ramos, C López, J Akerman, M Muñoz and J L Prieto 2015 Phys. Rev. B. 91, 214404

${ }^{14}$ P Ho, J Zhang, J A Currivan-Incordia, D C Bono and C A Ross 2015 IEEE Mag. Lett. 6, 3000104

${ }^{15}$ S-H Yang, K-S Ryu and S Parkin 2015 Nature Nanotechnology 10, 221
}

point. Some of the conclusions drawn in this work though have to be taken cautiously in experiments of DW depinning using materials with perpendicular magnetic anisotropy (PMA) ${ }^{29}$, where the domain wall movement is thermally activated.

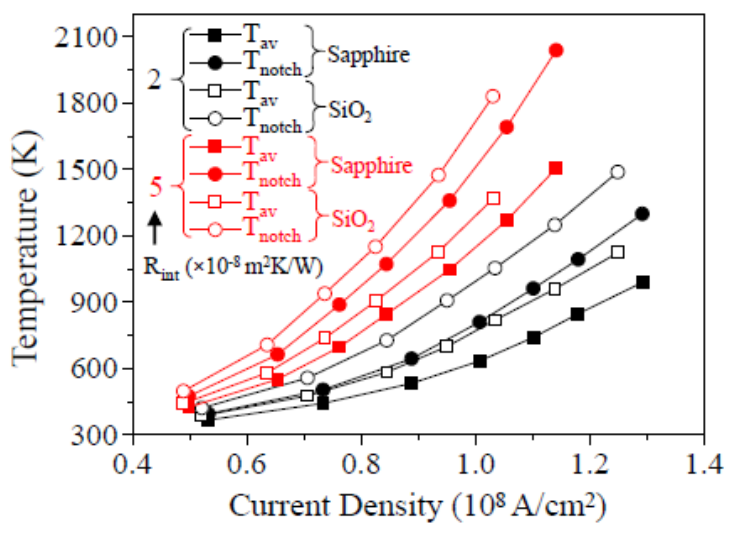

Figure 8. Average temperature in the nanostripe (squares) and temperature in the notch (circles) versus current density for a $100 \mathrm{~ns}$ pulse, calculated for devices deposited over Sapphire (filled symbols) or over $\mathrm{Si} / \mathrm{SiO}_{2}$ (open symbols) and for two different values of the thermal contact resistance, $2 \cdot 10^{-8}$ $\mathrm{m}^{2} \mathrm{~K} / \mathrm{W}$ (black) and $5 \cdot 10^{-8} \mathrm{~m}^{2} \mathrm{~K} / \mathrm{W}$ (red).

\section{Acknowledgements}

This work has been funded by the Spanish Ministerio de Economía y Competitividad through the projects MAT2014-52477-C5-1-P and MAT2014-52477-C5-3-P.

\footnotetext{
${ }^{16}$ S Emori, U Bauer, S-M Ahn and E Martínez 2013 Nat. Mater. 12, 611

${ }^{17}$ D W Lee and W d Kingery 1960 J. Am. Ceram. Soc. 43, 594

${ }^{18}$ K.-J Kim, J-C Lee, S-B Choe and K.-H. Shin 2008 Appl. Phys. Lett. 92, 192509

${ }^{19}$ C Y Ho, M W Ackerman, K Y Wu, T N Havill, R. H. Bogaard, R A Matula, S G Oh and H M James 1983 J. Phys. Chem. Ref. Data 12, 183

${ }^{20}$ S Moretti, V Raposo, E Martínez and L López-Díaz 2017 Phys. Rev. B 95, 064419

${ }^{21}$ One may use intrinsic $\mathrm{Si}$ substrate with native $\mathrm{SiO}_{2}$, but e-beam lithography on this substrate may be challenging. See also conclusions for an additional discussion on substrates that are good thermal conductor but electric insulators.

${ }^{22}$ See also Supplementary Information of Reference 5.

${ }^{23}$ G Nahrwold, L Bocklage, J M Scholtyssek, T Masuyama, B Krüger, U Merkt and G Meier 2009 J. Appl. Phys. 105, 07D511

${ }^{24}$ G Nahrwold, J M Scholtyssek, S Motl-Ziegler, O Albrecht, U Merkt and G Meier 2010 J. Appl. Phys. 108, 013907

${ }^{25}$ The slower rise time of the temperatura for larger thermal contact resistance can be intuitively understood by comparing the problem with the lumped heat approximation in unsteady state, which can be found in any textbook on heat transfer.

${ }^{26}$ J Akerman, M Muñoz, M Maicas and J L Prieto 2010 Phys. Rev. B 82, 064426

${ }^{27}$ M Muñoz and J L Prieto 2011 Nat. Commun. 2, 562

${ }^{28}$ D Castilla, M Maicas, J L Prieto and M Proenca 2017 J. Phys. D: Appl. Phys. 50, 105001

${ }^{29}$ M Cormier, A Mouguin, J Ferré, A Thiaville, N Charpentier, F Piéchon, R Weil, V Blatz and B Rodmacq 2010, Phys. Rev. B 82, 024407
} 